Exploration of trade-offs between steady-state and dynamic properties in signaling cycles

This article has been downloaded from IOPscience. Please scroll down to see the full text article.

2012 Phys. Biol. 9045010

(http://iopscience.iop.org/1478-3975/9/4/045010)

View the table of contents for this issue, or go to the journal homepage for more

Download details:

IP Address: 128.178.5.7

The article was downloaded on 15/08/2012 at 17:10

Please note that terms and conditions apply. 


\title{
Exploration of trade-offs between steady-state and dynamic properties in signaling cycles
}

\author{
A Radivojevic ${ }^{1,2}$, B Chachuat $^{3}$, D Bonvin ${ }^{2}$ and V Hatzimanikatis ${ }^{1}$ \\ ${ }^{1}$ Laboratory of Computational Systems Biotechnology, École Polytechnique Fédérale de Lausanne \\ (EPFL), Lausanne, Switzerland \\ ${ }^{2}$ Automatic Control Laboratory, École Polytechnique Fédérale de Lausanne (EPFL), Lausanne, \\ Switzerland \\ ${ }^{3}$ Centre for Process Systems Engineering (CPSE), Department of Chemical Engineering, Imperial \\ College London, London, UK \\ E-mail: vassily.hatzimanikatis@epfl.ch
}

Received 14 March 2012

Accepted for publication 22 May 2012

Published 7 August 2012

Online at stacks.iop.org/PhysBio/9/045010

\begin{abstract}
In the intracellular signaling networks that regulate important cell processes, the base pattern comprises the cycle of reversible phosphorylation of a protein, catalyzed by kinases and opposing phosphatases. Mathematical modeling and analysis have been used for gaining a better understanding of their functions and to capture the rules governing system behavior. Since biochemical parameters in signaling pathways are not easily accessible experimentally, it is necessary to explore possibilities for both steady-state and dynamic responses in these systems. While a number of studies have focused on analyzing these properties separately, it is necessary to take into account both of these responses simultaneously in order to be able to interpret a broader range of phenotypes. This paper investigates the trade-offs between optimal characteristics of both steady-state and dynamic responses. Following an inverse sensitivity analysis approach, we use systematic optimization methods to find the biochemical and biophysical parameters that simultaneously achieve optimal steady-state and dynamic performance. Remarkably, we find that even a single covalent modification cycle can simultaneously and robustly achieve high ultrasensitivity, high amplification and rapid signal transduction. We also find that the response rise and decay times can be modulated independently by varying the activating- and deactivating-enzyme-to-interconvertible-protein ratios.
\end{abstract}

S Online supplementary data available from stacks.iop.org/PhysBio/9/045010/mmedia

\section{Introduction}

In signaling networks, the basic units are covalent modification cycles, which comprise the activation and deactivation of proteins by other proteins. Protein modification in cell signaling-typically a phosphorylation and dephosphorylation-is a general mechanism responsible for the transfer of a wide variety of chemical signals in biological systems. Although the concept does not seem to be complex from a biochemical point of view, these simple systems can nevertheless provide a large diapason of dynamical responses and are therefore ubiquitous building blocks of signaling pathways. Mitogen-activated protein kinase cascades (MAPK) — an example of chains of interconvertible proteins-have been shown to drive many developmental processes and are also implicated in various diseases [1, 2].

Mathematical modeling and analysis of signaling units and networks have been used to understand the functions of these proteins (see [3-5] for review) and to study emergent 
phenomena, such as switch-like input-output responses $[6,7]$, bistability [8-12] and oscillatory behavior [13, 14]. A number of theoretical studies have elucidated some of the design principles of signaling networks and have proved useful for interpreting various experimental observations [15-20].

Parametric sensitivity analysis provides a powerful framework to help relate the structure to the function in complex networks. There exist two types of parametric sensitivity analyses, namely direct sensitivity analysis and inverse sensitivity analysis [21, 22]. These methods have been used for the analysis of both metabolic and signal transduction pathways. Direct sensitivity analysisthe consideration of changes in the system due to a variation in the model parameters-has been widely applied [23-28]. Inverse sensitivity analysis-the identification of the corresponding parameter values needed to achieve a desired functional behavior-has also been occasionally used in studies of metabolic networks to identify the relationship between model parameters and functions [29-31]. This second type involves solving constrained optimization problems and it is well adapted for studying biochemical networks, due to its ability to deal with large-scale models [32-34]. Moreover, the inverse approach leads to efficient parametric analysis and identification, contrary to an exhaustive parameter search.

In this paper, we investigate multiple steady-state and dynamic properties and their combinations using an inverse parametric sensitivity formulation. We consider covalent modification cycles with minimal number of degrees of freedom, and without assuming any feedback interaction or multiple phosphorylation occurrences. We identify the best combinations of model parameter values, achieving desired functional characteristics, such as high ultrasensitivity, low signal transduction time and high signal amplification. This approach is found to be particularly well suited for translating parameter values into design principles of signaling pathways. Furthermore, we account for inherent variability of cell components and discuss the optimal design for covalent modification cycles that are robust to such fluctuations.

\section{Materials and methods}

\section{Mechanistic modeling framework}

The system under consideration consists of three elements: (i) a kinase, (ii) an activating enzyme, which can be a receptor stimulated by a ligand or an activated kinase, and (iii) a deactivating enzyme, usually a phosphatase. The active receptor initiates the internal signaling cascade, including a series of protein phosphorylation state changes, which represents the basic unit in signal transduction networks.

The development of our mathematical models relies on the following assumptions that are commonly used in biochemical networks:

- the activation step involves the reversible binding of the activating enzyme to inactive kinase and the complex is irreversibly released;

- the inactivation step involves the reversible binding of the deactivating enzyme to active kinase and the complex is irreversibly released;

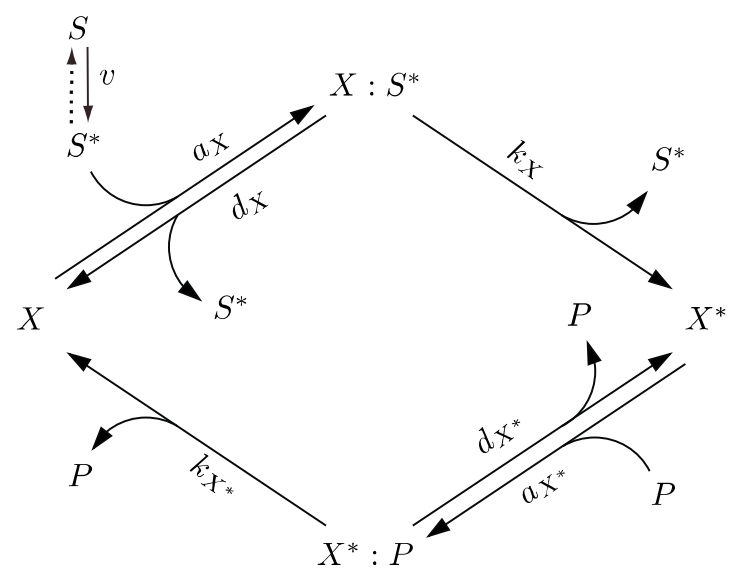

Figure 1. Schematic representation of a covalent modification cycle. A cycle is composed of two states of the same protein, namely the inactive state $X$ and the active state $X^{*}$. The activation and deactivation reactions are catalyzed by the enzymes $S^{*}$ and $P$, respectively.

- the total amount of kinase is taken to be constant for the signaling time scale considered;

- the rates of the various processes follow mass-action kinetics;

- the dynamics can be described by ordinary differential equations.

A commonly used simplification considers that the concentration in intermediate enzyme-substrate complex is small compared to the substrate concentrations and can therefore be neglected [6,10, 13], leading to simpler mathematical models. This simplification has been used mainly to reduce the computational effort or to obtain an exact analytical solution to the problem. However, it has been shown, and we found in our studies too, that if we neglect the so-called substrate sequestration in the form of intermediate enzymesubstrate complexes, the analysis may lead to incorrect model predictions [35-37]. Besides its effect on model prediction accuracy, substrate sequestration has been shown to induce both positive and negative feedback mechanisms in signaling cascades $[12,19]$. One key advantage of our computational approach is that it does not require such simplifications and, accordingly, substrate sequestration is fully considered here.

\section{Mathematical models}

The covalent modification cycle represented in figure 1 comprises seven species $S, S^{*}, X, X^{*}, P, X: S^{*}$ and $X^{*}: P$. The receptor changes its own state from susceptible $S$ to active $S^{*}$. Since our focus is on the dynamics of the internal module, the activation/deactivation of the receptor is assumed to occur under very fast kinetics. The kinetic mechanism and the initial conditions for the system are detailed in the appendix. The variables $X$ and $X^{*}$ represent the two interconvertible forms of one protein, e.g. the phosphorylated and dephosphorylated forms of a kinase. The proteins $S^{*}$ and $P$ catalyze the activation and deactivation reactions and are both referred to as enzymes subsequently. The activation steps proceed through the formation of intermediate enzyme-substrate complexes $X: S^{*}$ and $X^{*}: P$. The enzyme $S^{*}$ can be either the activating 
Table 1. Dimensionless variables and parameters in the dynamic model (7)-(12).

\begin{tabular}{|c|c|c|c|}
\hline Concentrations & & & \\
\hline $\begin{array}{l}\text { Inactive and active forms of interconvertible } \\
\text { protein }\end{array}$ & $x:=\frac{[X]}{\left[X_{T}\right]}$ & $x^{*}:=\frac{\left[X^{*}\right]}{\left[X_{T}\right]}$ & \\
\hline $\begin{array}{l}\text { Inactive and active forms of signaling enzyme, } \\
\text { and enzyme-substrate complex }\end{array}$ & $s:=\frac{[S]}{\left[S_{T}\right]}$ & $s^{*}:=\frac{\left[S^{*}\right]}{\left[S_{T}\right]}$ & $\left\{x: s^{*}\right\}:=\frac{\left[X: S^{*}\right]}{\left[S_{T}\right]}$ \\
\hline $\begin{array}{l}\text { Phosphatase, and phosphatase-substrate complex } \\
\text { Kinetic parameters }\end{array}$ & $p:=\frac{[P]}{\left[P_{T}\right]}$ & & $\left\{x^{*}: p\right\}:=\frac{\left[X^{*}: P\right]}{\left[P_{T}\right]}$ \\
\hline Complex dissociation rate constants & $\tilde{d}_{X}:=\frac{d_{X}}{k_{X^{*}}}$ & $\tilde{d}_{X^{*}}:=\frac{d_{X^{*}}}{k_{X^{*}}}$ & $\tilde{k}_{X}:=\frac{k_{X}}{k_{X^{*}}}$ \\
\hline Complex formation rate constants & $\tilde{a}_{X}:=\frac{a_{X}}{k_{X^{*}}}\left[X_{T}\right]$ & $\tilde{a}_{X^{*}}:=\frac{a_{X^{*}}}{k_{X^{*}}}\left[X_{T}\right]$ & \\
\hline $\begin{array}{l}\text { Concentration ratios } \\
\text { Input signaling enzyme to interconvertible } \\
\text { protein, and phosphatase to interconvertible } \\
\text { protein }\end{array}$ & $\rho^{S / X}:=\frac{\left[S_{T}\right]}{\left[X_{T}\right]}$ & $\rho^{P / X}:=\frac{\left[P_{T}\right]}{\left[X_{T}\right]}$ & \\
\hline Time & $\tau:=k_{X^{*}} t$ & & \\
\hline
\end{tabular}

kinase of an upstream cycle or the receptor responding to an external input signal (e.g. growth factor or hormone level), while the enzyme $P$ corresponds to a phosphatase.

The total amount of interconvertible protein $X_{T}$, as well as the total amounts of the activation/deactivation proteins $S_{T}$ and $P_{T}$, is considered to be constant in the time scale considered. The corresponding conservation equations relating the concentrations of the seven species read

$$
\begin{aligned}
& {\left[X_{T}\right]=[X]+\left[X^{*}\right]+\left[X: S^{*}\right]+\left[X^{*}: P\right],} \\
& \text { with } \frac{\mathrm{d}\left[X_{T}\right]}{\mathrm{d} t}=0, \\
& {\left[S_{T}\right]=[S]+\left[S^{*}\right]+\left[X: S^{*}\right], \quad \text { with } \quad \frac{\mathrm{d}\left[S_{T}\right]}{\mathrm{d} t}=0,} \\
& {\left[P_{T}\right]=[P]+\left[X^{*}: P\right], \quad \text { with } \quad \frac{\mathrm{d}\left[P_{T}\right]}{\mathrm{d} t}=0 .}
\end{aligned}
$$

The conservation laws (1)-(3) relate the concentrations of the species in the system. Three additional (independent) relations can be obtained by writing the mass-balance equations for $X^{*}$, $X: S^{*}$ and $X^{*}: P$ as

$$
\begin{gathered}
\frac{\mathrm{d}\left[X^{*}\right]}{\mathrm{d} t}=-a_{X^{*}}\left[X^{*}\right][P]+k_{X}\left[X: S^{*}\right]+d_{X}\left[X^{*}: P\right], \\
\frac{\mathrm{d}\left[X: S^{*}\right]}{\mathrm{d} t}=a_{X}[X]\left[S^{*}\right]-\left(d_{X}+k_{X}\right)\left[X: S^{*}\right], \\
\frac{\mathrm{d}\left[X^{*}: P\right]}{\mathrm{d} t}=a_{X^{*}}\left[X^{*}\right][P]-\left(d_{X^{*}}+k_{X^{*}}\right)\left[X^{*}: P\right],
\end{gathered}
$$

where $a_{X}, d_{X}, k_{X}, a_{X^{*}}, d_{X^{*}}$ and $k_{X^{*}}$ are the parameters of the mass-action kinetic laws, as shown in figure 1.

The basic structure of signaling cycles is well conserved in cells, even though it can generate a high variety of biological responses. In order to facilitate the discovery of such general features, it is useful to consider dimensionless parameters and variables, rather than focussing on a particular system [6]. Table 1 gives the dimensionless variables and parameters used to conduct the analysis. The following dimensionless set of differential algebraic equations (DAEs) is obtained:

$$
\begin{gathered}
\frac{\mathrm{d} x^{*}}{\mathrm{~d} \tau}=\rho^{S / X} \widetilde{k}_{X}\left\{x: s^{*}\right\}+\rho^{P / X}\left({\widetilde{d_{X}}}_{X^{*}}\left\{x^{*}: p\right\}-\widetilde{a}_{X^{*}} x^{*} p\right) \\
\frac{\mathrm{d}\left\{x: s^{*}\right\}}{\mathrm{d} \tau}=\widetilde{a}_{X} x s^{*}-\left(\widetilde{d}_{X}+\widetilde{k}_{X}\right)\left\{x: s^{*}\right\}
\end{gathered}
$$

$$
\begin{gathered}
\frac{\mathrm{d}\left[x^{*}: p\right]}{\mathrm{d} \tau}=\tilde{a}_{X^{*}} x^{*} p-\left(\tilde{d}_{X^{*}}+1\right)\left\{x^{*}: p\right\} \\
1=x+x^{*}+\rho^{S / X}\left\{x: s^{*}\right\}+\rho^{P / X}\left\{x^{*}: p\right\} \\
1=s+s^{*}+\left\{x: s^{*}\right\} \\
1=p+\left\{x^{*}: p\right\} .
\end{gathered}
$$

When the system is at steady state and assuming that all of signaling enzyme is in its active form $\bar{s}=0$, the dynamic model (7)-(12) reduces to the following set of algebraic equations:

$$
\begin{gathered}
0=\overline{\left\{x: s^{*}\right\}}-\frac{\bar{x}}{\widetilde{K}_{X}+\bar{x}} \\
0=\overline{\left\{x^{*}: p\right\}}-\frac{\overline{x^{*}}}{\widetilde{K}_{X^{*}}+\overline{x^{*}}} \\
0=\overline{\left\{x^{*}: p\right\}}-\alpha_{X} \overline{\left\{x: s^{*}\right\}} \\
1=\bar{x}+\overline{x^{*}}+\rho^{S / X} \overline{\left\{x: s^{*}\right\}}+\rho^{P / X} \overline{\left\{x^{*}: p\right\}} \\
1=\overline{s^{*}}+\overline{\left\{x: s^{*}\right\}} \\
1=\bar{p}+\overline{\left\{x^{*}: p\right\}},
\end{gathered}
$$

where $(\bar{*})$ indicates a variable at steady state, and $\widetilde{K}_{X}=\frac{\widetilde{a}_{X}+\widetilde{k}_{X}}{\tilde{d}_{X}}$, $\widetilde{K}_{X^{*}}=\frac{\widetilde{a}_{X^{*}}+1}{\widetilde{d}_{X^{*}}}$ and $\alpha_{X}=\widetilde{k}_{X} \frac{\rho^{S / X}}{\rho^{P / X}}$ are the new parameters.

\section{Design parameters}

The foregoing steady-state formulation (13)-(18) identifies two dimensionless Michaelis-Menten constants $\widetilde{K}_{X}$ and $\widetilde{K}_{X^{*}}$, which have been shown to determine the most important features of steady-state responses. They are in turn nonlinear combinations of the dimensionless kinetic rates $\tilde{a}_{X}, \widetilde{a}_{X^{*}}, \widetilde{d}_{X}, \widetilde{d}_{X^{*}}, \widetilde{k}_{X}$, and thus provide the link between steadystate and dynamic responses.

Furthermore, the parameter $\alpha_{X}$ represents the activation potential of the cycle and describes the balance between activation and deactivation of the kinase. It has been shown that in the special case, when all the corresponding parameters of the upper and lower branch in the cycle are the same and $\alpha_{X}=1$, the system is at or very close to its inflection point [6]. In the formulation above, we have included the balance of the first signaling input (in this case the receptor) in order to capture changes in the activating input due to changes in protein amount. 
(a)

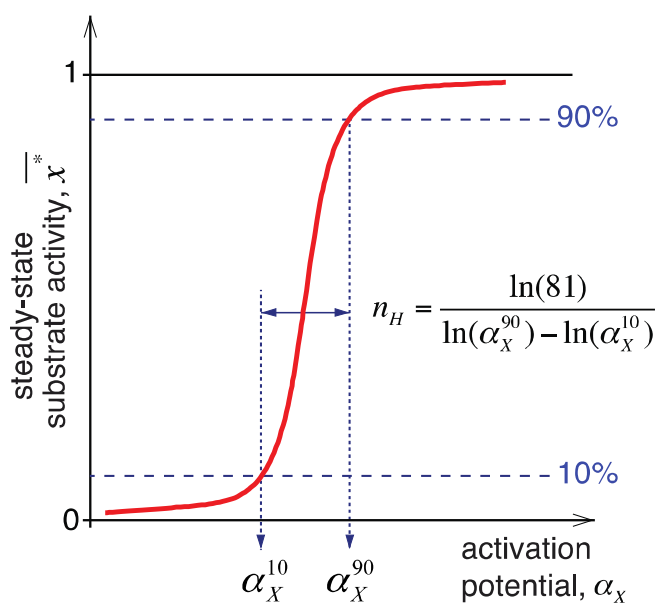

(c)

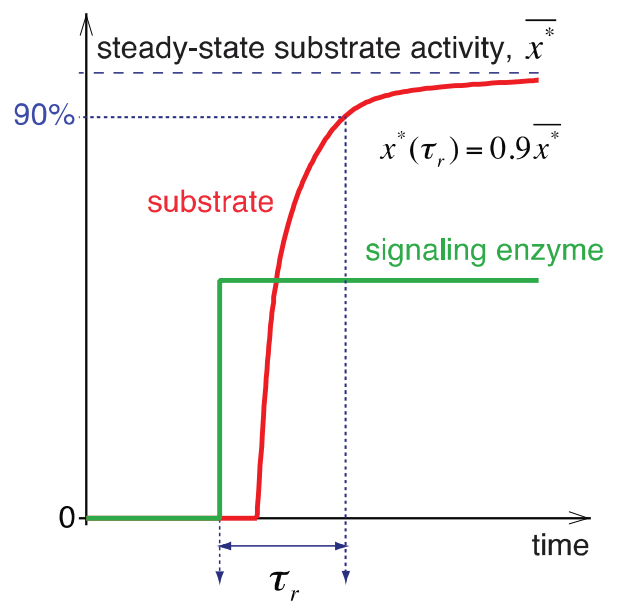

(b)

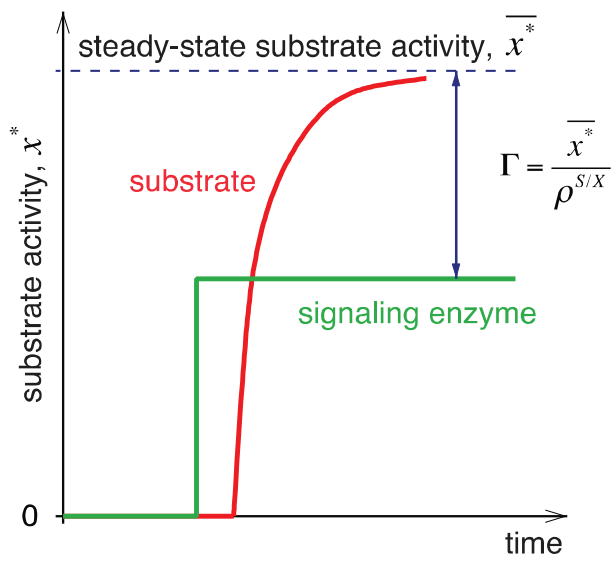

(d)

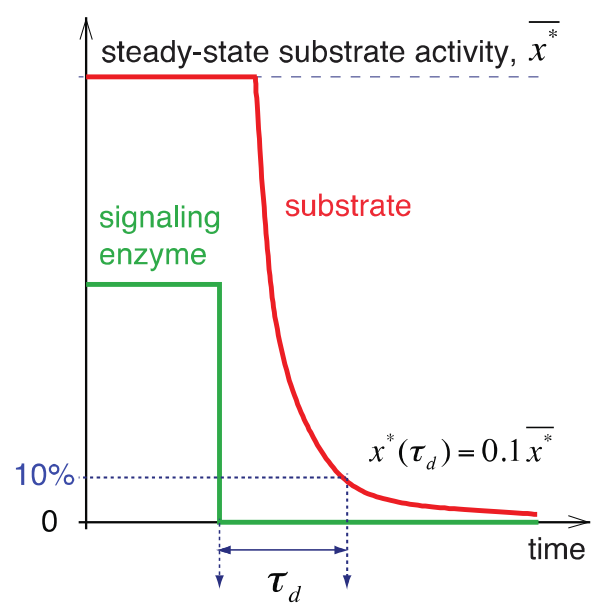

Figure 2. Design criteria. (a) The ultrasensitivity coefficient $n_{H}$ provides a measure of the sensitivity of substrate activity to changes in input signal activity, at steady state. $(b)$ The amplification gain $\Gamma$ is a measure of the relative strength of the response to a given input level, at steady state. (c) The rise time $\tau_{r}$ is a measure of the speed at which a stimulation signal is transduced. (d) The decay time $\tau_{d}$ provides a measure of the speed at which an activated state vanishes upon canceling the stimulation signal.

\section{Design criteria}

Several criteria have been considered to assess signal transduction properties. Ultrasensitivity (figure $2(a)$ ) and gain amplification (figure $2(b)$ ) are steady-state criteria, whereas rise time (figure $2(c)$ ), decay time (figure $2(d)$ ) and signal duration are transient criteria. Since the last criterion is more relevant in the analysis in the study of signaling cascades, we do not consider it in this study.

Ultrasensitivity. Signaling pathways with ultrasensitive input-output characteristics convert gradual changes in all or nothing type decisions. This property is defined as the response of a system that is more sensitive to the input concentration than a normal hyperbolic Michaelis-Menten response [6]. For instance, to increase the reaction rate 9 -fold from $10 \%$ to $90 \%$ of the maximum activation, a typical Michaelis-Menten response requires 81 -fold increase in input concentration. An ultrasensitive response should need less ligand concentration change to accomplish the same. The degree of ultrasensitivity depends on the size of the input window within which the response changes from nothing to all.
The ability of signaling cycles to produce ultrasensitive response has been observed experimentally, and it has been suggested that many physiological phenomena such as cancer progression and morphogenesis are associated with this feature (see, e.g., [7]). Several mechanisms can lead to switch-like stimulus-response curves, examples of which are the cooperative allosteric effect of multisite protein [38], saturation of enzymes [6] and positive feedback [9]. When combined with negative feedback, ultrasensitive modules can lead to oscillations [13], but these modules also add robustness against stochastic fluctuations [39].

Goldbeter and Koshland [6] introduced a measure of ultrasensitivity in signaling cycles based on the similarity of the ultrasensitive response with the sigmoidal (Hill) kinetics of allosteric proteins. They quantified ultrasensitivity by the apparent Hill coefficient $n_{H}$ of the output response as

$$
n_{H}=\frac{\ln (81)}{\ln \left(\alpha_{X}^{90}\right)-\left(\alpha_{X}^{10}\right)},
$$

where the variables $\alpha_{X}^{10}$ and $\alpha_{X}^{90}$ are the activation potentials of the signaling kinase required to achieve $10 \%$ and 
$90 \%$, respectively, of the maximal activation of the output (figure 2(a)),

$$
\begin{aligned}
& \alpha_{X}^{10}: \overline{x^{*}}\left(\alpha_{X}^{10}\right)=0.1 \lim _{\alpha_{X} \rightarrow \infty} \overline{x^{*}}\left(\alpha_{X}\right) \\
& \alpha_{X}^{90}: \overline{x^{*}}\left(\alpha_{X}^{90}\right)=0.9 \lim _{\alpha_{X} \rightarrow \infty} \overline{x^{*}}\left(\alpha_{X}\right) .
\end{aligned}
$$

In general, this measure does not require the response curve to be point symmetric, and the larger the apparent Hill coefficient in (19), the more ultrasensitive the system.

Determining the apparent Hill coefficients in complex signaling cycles can be computationally demanding, especially considering that the study of design criteria involves exhaustive parameter search. To facilitate this computation, it is advantageous to transform the steady-state model (13)(18) into a set of DAEs in $\alpha_{X}$-see model (A.3)-(A.8) in the appendix. This way, the values of $\alpha_{X}^{10}$ and $\alpha_{X}^{90}$ defined implicitly via relations (20) and (21) can be obtained using numerical continuation [40] and well-established event detection techniques in DAEs [41].

Amplification. The amplification gain in signaling cycles defines a measure of response strength. It is a relevant biological characteristic because the response produced by a signaling cycle must exceed a certain magnitude in order to trigger downstream reactions. However, a very large amplification may not always be warranted for a signal to be transmitted to its final target.

The amplification gain is defined as the ratio of activated substrate concentration to input concentration at steady state. We consider that the relevant input concentration accounts for the active form of the receptor, either in free or complexed form, thus giving the definition of a dimensionless amplification gain,

$$
\Gamma:=\frac{\overline{x^{*}}}{\rho^{S / X}},
$$

where $\overline{x^{*}}$ stands for the steady-state substrate activity corresponding to a given level of signaling enzyme (figure 2(b)). From this definition, it is clear that higher amplification gains are favored by the smaller signaling enzyme-to-substrate ratios, $\rho^{S / X} \ll 1$. This also explains why those models ignoring intermediate complex formation tend to overestimate amplification (see [6]).

Signaling times. The time needed for an output signal to reach a certain threshold with respect to a reference state in response to an input signal is an important characteristic of signaling cycles and, more generally, of signal transduction pathways. It has been proposed that short signaling time is a desirable characteristic in various biological pathways [15, 42]. Here, we consider two different signaling times.

- The rise time $\tau_{r}$ is a measure of how fast an activation signal propagates through a cycle. We specifically define $\tau_{r}$ as the time needed to reach $90 \%$ of the steady-state substrate activation in response to sustained step activation of the input, starting from the reference (inactive) state (figure 2(c)):

$$
\tau_{r}: x^{*}\left(\tau_{r}\right)=0.9 \overline{x^{*}}, \text { with } x^{*}(0)=0 .
$$

Note that this definition of signaling time differs from the one in [15], which considers the average time needed to activate the substrate. This latter measure would be inappropriate here, as the activation time grows unbounded in the case of a permanently activated pathway.

- The decay time $\tau_{d}$ is the time needed for the substrate activity of interconvertible kinase, starting from the steady-state activation level, to decrease to within $10 \%$ of this initial activity after the stimulus has been ceased (figure $2 d$ ):

$$
\tau_{d}: x^{*}\left(\tau_{d}\right)=0.1 \overline{x^{*}}, \text { with } x^{*}(0)=\overline{x^{*}} .
$$

The above definitions assume step inputs. Alternatively, one could also consider exponential, impulse or rectangular inputs, although this would require redefining (23) and (24).

\section{Results and discussion}

\section{Optimal design for ultrasensitivity}

The ultrasensitivity of covalent modification cycles has been studied extensively since its discovery by Goldbeter and Koshland [6]. Their classical results suggest that a monocyclic cascade can display ultrasensitive responses even though the interconversion steps follow Michaelis-Menten kinetics. Since then, a number of studies have elaborated further on these results [43-45].

In this subsection, we apply the inverse sensitivity analysis in order to identify the subdomains of the parameter space that lead to ultrasensitive responses. We vary the dimensionless Michaelis-Menten constants $\widetilde{K}_{X}, \widetilde{K}_{X^{*}}$ and the concentration ratios $\rho^{S / X}, \rho^{P / X}$ and measure how the input signal $s^{*}$ affects the relative concentration of protein in active state $x^{*}$ based on the steady-state model (A.3)-(A.8).

Figure 3 illustrates how ultrasensitivity is determined by the values of $\widetilde{K}_{X}$ and $\widetilde{K}_{X^{*}}$ for fixed values of $\rho^{S / X}$ and $\rho^{P / X}$. This behavior is in good agreement with the main conclusions in [6], namely

- ultrasensitivity is promoted by low values of the dimensionless Michaelis-Menten constants $\widetilde{K}_{X}$ and $\widetilde{K}_{X^{*}}$;

- ultrasensitivity is only possible when the total concentration in activating enzyme $\left[S_{T}\right]$ and the total concentration in deactivating enzyme $\left[P_{T}\right]$ are small compared to the total concentration in interconvertible protein $\left[X_{T}\right]$, i.e. $\rho^{S / X}$ and $\rho^{P / X} \ll 1$;

- the stimulus response is point symmetric when $\widetilde{K}_{X}=\widetilde{K}_{X^{*}}$ and $\rho^{S / X}, \rho^{P / X} \rightarrow 0$.

Next, we consider the inverse sensitivity approach and solve the following optimization problem in order to determine optimal values of the dimensionless MichaelisMenten constants $\widetilde{K}_{X}$ and $\widetilde{K}_{X^{*}}$,

$$
\begin{array}{ll}
\text { find } & \widetilde{K}_{X}, \widetilde{K}_{X^{*}} \text { that } \\
\text { maximize } & n_{H}(19) \\
\text { subject to } & \text { steady-state model (A.3)-(A.8). }
\end{array}
$$

The parameters $\widetilde{K}_{X}$ and $\widetilde{K}_{X^{*}}$ are taken in the range $\left[10^{-2}, 10^{2}\right]$, which was found to be wide enough from comparisons with 

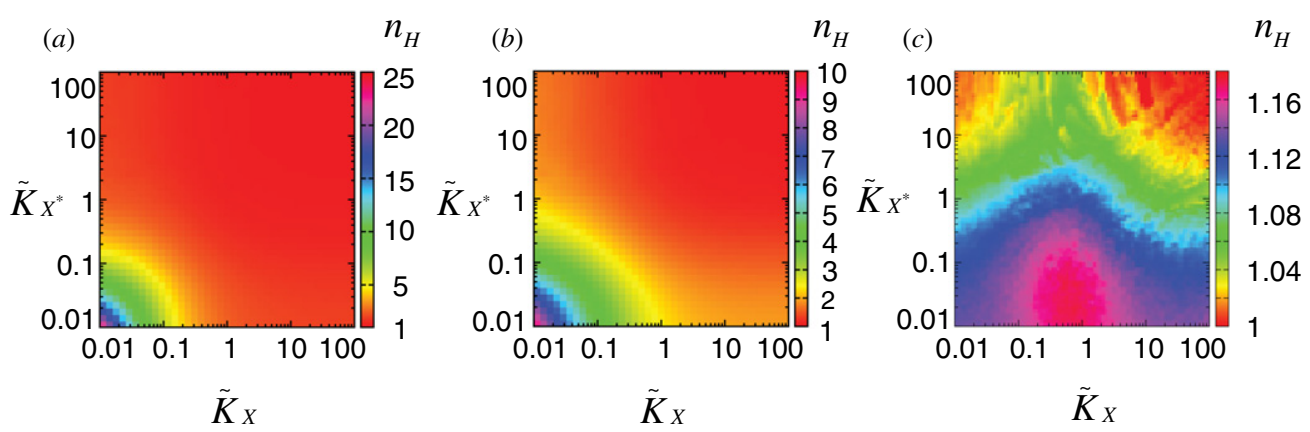

Figure 3. Landscape of Hill coefficient value $n_{H}$ versus dimensionless Michaelis-Menten constants $\widetilde{K}_{X}$ and $\widetilde{K}_{X^{*}}$ for the three different values of concentration ratios: $(a) \rho^{S / X}=\rho^{P / X}=10^{-2},(b) \rho^{S / X}=\rho^{P / X}=10^{-1},(c) \rho^{S / X}=\rho^{P / X}=1$.
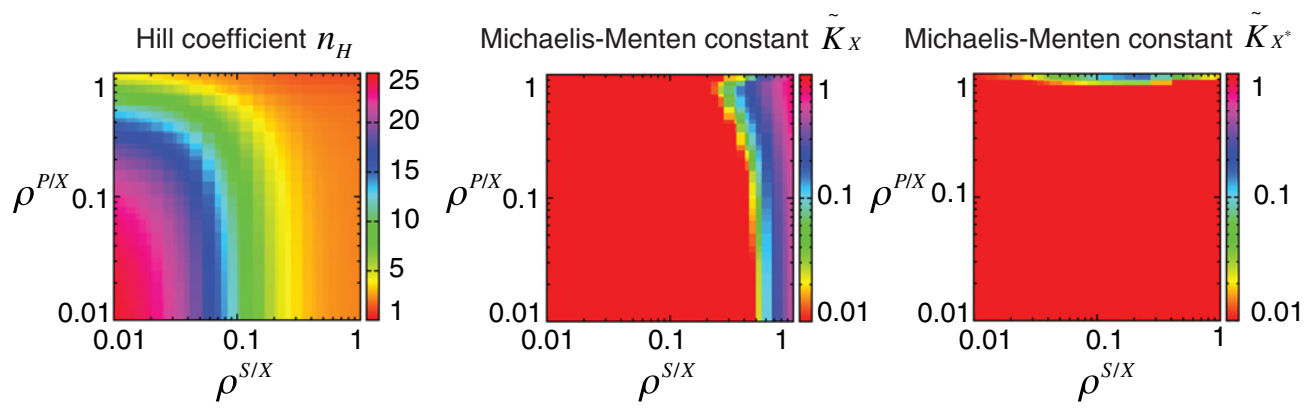

Figure 4. Design for maximal ultrasensitivity. The optimal Hill coefficient and Michaelis-Menten constants are computed by solving the optimization problem $\left(\mathrm{P}_{1}\right)$, for values of the concentration ratios $\rho^{S / X}$ and $\rho^{P / X}$ in the range $\left[10^{-2}, 1\right]$. The largest possible Hill coefficient values $n_{H}$ (left plot), with the corresponding dimensionless Michaelis-Menten constant $K_{X}$ (middle plot) and the corresponding dimensionless Michaelis-Menten constant $\widetilde{K}_{X^{*}}$ (right plot), are displayed.

larger parameter ranges. Moreover, following the observations in figure 3 , the concentration ratios $\rho^{S / X}$ and $\rho^{P / X}$ are taken in the range $\left[10^{-2}, 1\right]$.

The results shown in figure 4 lead to the following observations.

- Significant ultrasensitivity $\left(n_{H}>6\right)$ can only be achieved (i) for values of the dimensionless Michaelis-Menten constants $\widetilde{K}_{X}$ and $\widetilde{K}_{X^{*}}$ lower than $10^{-1}$ and (ii) for small values of the two concentration ratios $\rho^{S / X}, \rho^{P / X} \ll 1$.

- Ultrasensitivity can accommodate higher phosphatase levels $\left(\rho^{P / X}<0.5\right)$ than activating enzyme levels $\left(\rho^{S / X}<\right.$ $0.1)$.

The first observation is not a new result for it was suggested as a design criterion for ultrasensitivity already by [6]. On the other hand, the interplay and relative effect of the activation and deactivation steps constitute a new observation that follows from the proposed optimization methodology. In particular, this result also suggests that overexpression in activating proteins, such as receptors relative to target kinases, might lead to (pathological) loss of ultrasensitivity.

\section{Optimal design for signaling times under the amplification constraint}

In this subsection, we follow the inverse approach and formulate an optimization problem to identify the kinetic parameter values that minimize the response time, while at the same time satisfying a given amplification level $\Gamma$. Separate formulations are considered for the rise time $\tau_{r}\left(\mathrm{P}_{2}\right)$ and for the decay time $\tau_{d}\left(\mathrm{P}_{3}\right)$,

find $\quad \tilde{a}_{X}, \tilde{a}_{X^{*}}, \tilde{d}_{X}, \tilde{d}_{X^{*}}, \tilde{k}_{X}$ that

minimize rise time $\tau_{r}(23)$

subject to amplification gain $\Gamma(22)$ transient model (7)-(12),

and

find $\quad \tilde{a}_{X}, \tilde{a}_{X^{*}}, \tilde{d}_{X}, \tilde{d}_{X^{*}}, \tilde{k}_{X}$ that

minimize decay time $\tau_{d}$ (24)

subject to amplification gain $\Gamma$ (22)

transient model (7)-(12).

The model parameters $\widetilde{a}_{X}, \widetilde{a}_{X^{*}}, \tilde{d}_{X}, \widetilde{d}_{X^{*}}, \widetilde{k}_{X}$ are all taken in the range $\left[10^{-3}, 10^{3}\right]$ subsequently. Recall that model (7)(12) was made dimensionless, and so a kinetic parameter whose value is either at its lower bound $10^{-3}$ or at its upper bound $10^{3}$ reflects a very small or very large value relative to other parameters. To confirm that this parameter range is large enough to reveal the actual set of behaviors, we performed similar computations with wider parameter ranges as $\left[10^{-5}, 10^{5}\right]$ (see figure $\mathrm{S} 1$ in the supporting material available at stacks.iop.org/PhysBio/9/045010/mmedia). It was found that the general trend is conserved in that the parameters that were at their lower/upper bounds remained at their bounds, and those taking intermediate values also remained intermediate; moreover, optimization with wider parameter ranges only leads to marginal improvements in signaling times. We also performed additional computations with more narrow 

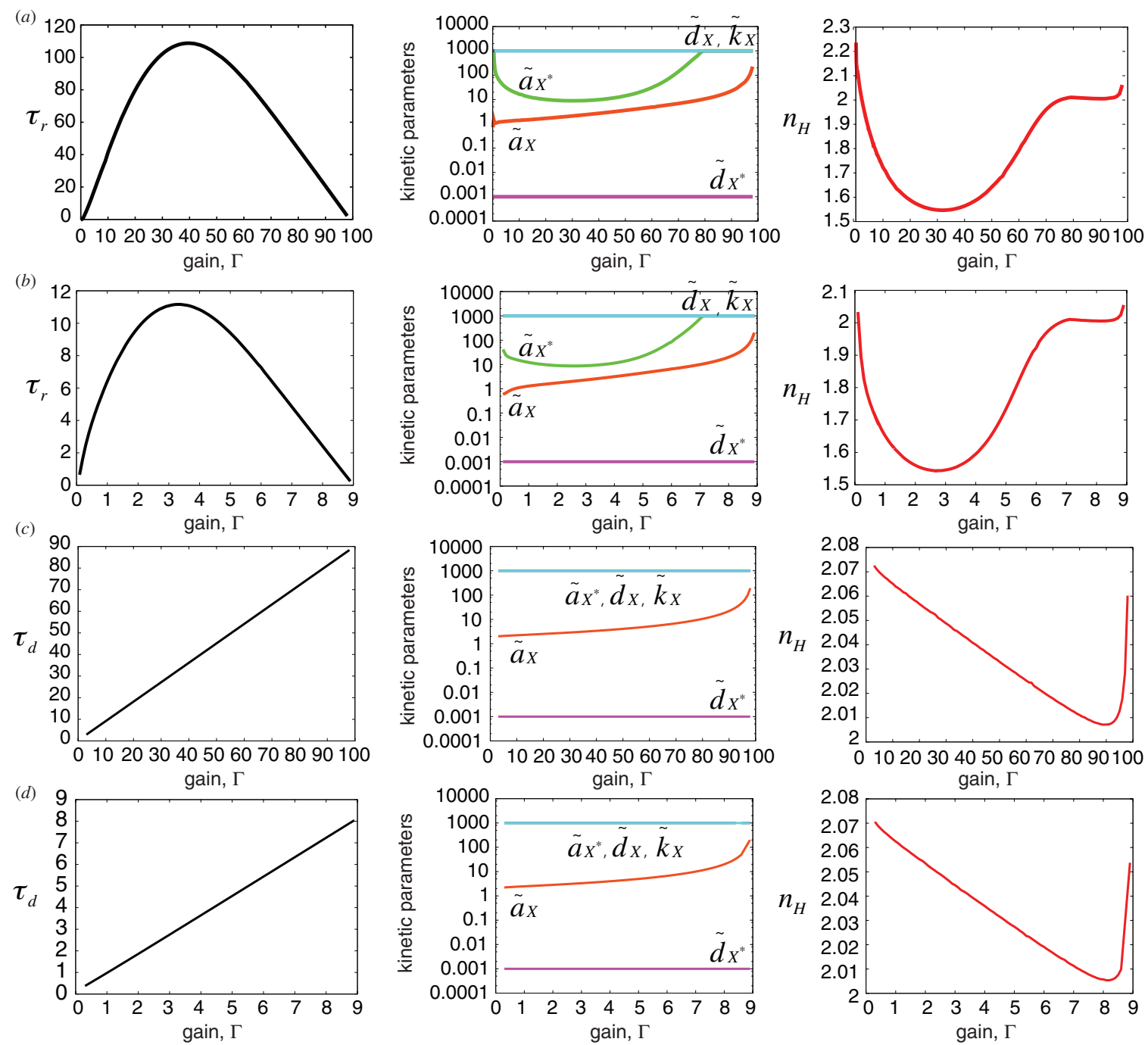

Figure 5. Design for minimal signaling times subject to given amplification. Optimal rise time $\tau_{r}(a$ and $b)$ and decay time $\tau_{d}(c$ and $d$ ), corresponding kinetic parameters $\widetilde{a}_{X}, \widetilde{a}_{X^{*}}, \widetilde{d}_{X}, \widetilde{d}_{X^{*}}, \widetilde{k}_{X}$ (middle plots) and Hill coefficient $n_{H}$ (right plots) versus amplification gain, for the concentration ratio regime $\rho^{S / X}=\rho^{P / X}=10^{-2}(a$ and $c)$ and concentration ratio regime $\rho^{S / X}=\rho^{P / X}=10^{-1}(b$ and $d)$.

parameter ranges as $\left[10^{-2}, 10^{2}\right]$, which again led to essentially the same trade-offs and minor differences in signaling times.

Figure 5 (left plots) displays the optimal signaling times as a function of the amplification gain $\Gamma$, for two different regimes of concentration ratios $\rho^{S / X}=\rho^{P / X}=10^{-2}$ and $\rho^{S / X}=\rho^{P / X}=10^{-1}$. Qualitatively, the optimization results appear to be very similar in both regimes. The feasible range of amplification gains is much wider with the lower values of concentration ratios - as expected from the relative gain definition in (22). The rise time $\tau_{r}$ (figures $5(a)$ and $(b)$ ) first increases monotonically with $\Gamma$ for low amplification. Beyond a certain amplification threshold, the rise time then decreases monotonically with $\Gamma$, thus indicating that the system can respond faster while achieving higher levels of amplification. This amplification threshold depends on the concentration ratios $\rho^{S / X}$ and $\rho^{P / X}$; it is close to $\Gamma \approx 40$ in the operating regime $\rho^{S / X}=\rho^{P / X}=10^{-2}$ (figure $5(a)$ ) and around $\Gamma \approx$ 3.3 in the regime $\rho^{S / X}=\rho^{P / X}=10^{-1}$ (figure 5(b)). On the other hand, the decay time $\tau_{d}$ (figures $5(c)$ and $(d)$ ) increases linearly with the amplification gain $\Gamma$. This is due to the fact that higher amplification leads to higher concentrations in activated protein form, which in turn takes more time to return to inactive state. Moreover, higher concentration ratios $\rho^{S / X}$ and $\rho^{P / X}$ lead to lower maximal possible levels of free active protein and, consequently, decay times are almost ten times shorter in the regime $\rho^{S / X}=\rho^{P / X}=10^{-1}$ compared to the regime $\rho^{S / X}=\rho^{P / X}=10^{-2}$ (figures $5(b),(d)$ versus $(a),(c)$ ). Such nonlinear relationships between various design criteria demonstrate the need for systematic optimization methods to analyze signaling pathways in a comprehensive manner.

In order to better understand these relationships, figure 5 (middle plots) displays the optimal kinetic parameter values $\widetilde{a}_{X}, \widetilde{a}_{X^{*}}, \widetilde{d}_{X}, \widetilde{d}_{X^{*}}, \widetilde{k}_{X}$ leading to minimal signaling times as a function of the amplification gain $\Gamma$. Both dissociation rate constants $\widetilde{d}_{X}$ and $\widetilde{k}_{X}$ of the kinase complex $X: S^{*}$ into $x$ and $x^{*}$, respectively, stay at their upper bounds and the rate constant $\widetilde{d}_{X^{*}}$ of the phosphatase complex $X^{*}: P$ into $x^{*}$ remains at its lower bound in all cases and regardless of the amplification level $\Gamma$. Particularly counter-intuitive is the finding that minimum response times are not achieved when 
the rate of formation $\tilde{a}_{X}$ of the kinase complex $X: S^{*}$ is maximum, which suggests that faster signal propagation with amplification is promoted by a more unstable complex $X: S^{*}$. These findings are in good agreement with the computational results for a covalent modification cycle obtained in [34]. It is also observed that higher levels of amplification together with shorter rise and decay times are achieved for an increasing value of the complex formation rate constant $\tilde{a}_{X}$, which suggests that $\widetilde{a}_{X}$ is the primary determining parameter for minimal signaling times under amplification constraints.

Nevertheless, signaling cycles so designed do not promote high ultrasensitivity as seen from figure 5 (right plots). This is because the constraints imposed on the kinetic parameters correspond to the values of $\widetilde{K}_{X}$ and $\widetilde{K}_{X^{*}}$ at which the Hill coefficient is no larger than $n_{H} \approx 2$. It is therefore critical to take the ultrasensitivity criterion into account simultaneously with the response time and amplification criteria in analyzing the design of signaling cycles.

\section{Optimal design for signaling times under amplification and ultrasensitivity constraints}

The ability of monocyclic cascades to achieve a high Hill coefficient for small values of the Michaelis-Menten constants is one of the most basic findings since their early study in the 1980s [6]. But with such a design, the system may exhibit excessively long response times as well as low signal amplification. In this subsection, we investigate whether a simple covalent modification cycle can simultaneously achieve fast signaling, high amplification and high ultrasensitivity.

A previous analysis has underpinned the strong dependence of ultrasensitivity with respect to the parameters $\widetilde{K}_{X}$ and $\widetilde{K}_{X^{*}}$, which are themselves functions of the dynamic model parameters,

$$
\frac{\widetilde{d}_{X}+\tilde{k}_{X}}{\widetilde{a}_{X}}=\widetilde{K}_{X} \quad \text { and } \quad \frac{\widetilde{d}_{X^{*}}+1}{\widetilde{a}_{X^{*}}}=\widetilde{K}_{X^{*}} .
$$

Incorporating an ultrasensitivity objective in the optimization problems $\left(\mathrm{P}_{2}\right)$ and $\left(\mathrm{P}_{3}\right)$ can be done in either one of the two ways.

(1) Optimize the steady-state and transient kinetic parameters jointly and enforce constraints (25) directly. This requires accounting for both the transient model (7)-(12) and the steady-state model (A.3)-(A.8) in the optimization problem.

(2) Optimize the transient kinetic parameters only, while enforcing an ultrasensitivity target indirectly via fixing the Michaelis-Menten constants to the values $\widetilde{\kappa}_{X}, \widetilde{\kappa}_{X^{*}}$ as

$$
\begin{gathered}
\widetilde{d}_{X}+\widetilde{k}_{X}-\widetilde{\kappa}_{X} \widetilde{a}_{X}=0 \\
\widetilde{d}_{X^{*}}+1-\widetilde{\kappa}_{X^{*}} \widetilde{a}_{X^{*}}=0 .
\end{gathered}
$$

This no longer requires the steady-state model (A.3)-(A.8) in the optimization problem and, besides, the foregoing constraints (26) and (27) are linear.

The second approach is considered next. Note that there remains flexibility in the choice of the kinetic parameters after enforcing constraints (26) and (27) - three remaining degrees of freedom out of five, thereby leaving significant freedom for optimization. As previously, separate optimization formulations are considered for minimizing the rise time $\tau_{r}\left(\mathrm{P}_{4}\right)$ and the decay time $\tau_{d}\left(\mathrm{P}_{5}\right)$,

$$
\begin{aligned}
& \text { find } \quad \tilde{a}_{X}, \tilde{a}_{X^{*}}, \tilde{d}_{X}, \tilde{d}_{X^{*}}, \tilde{k}_{X} \text { that } \\
& \text { minimize rise time } \tau_{r} \text { (23) } \\
& \text { subject to amplification gain } \Gamma \text { (22) } \\
& \text { target } n_{H}(26)-(27) \\
& \text { transient model }(7)-(12)
\end{aligned}
$$

and

$$
\begin{array}{ll}
\text { find } & \tilde{a}_{X}, \tilde{a}_{X^{*}}, \tilde{d}_{X}, \tilde{d}_{X^{*}}, \tilde{k}_{X} \\
\text { minimize } & \text { decay time } \tau_{d}(24) \\
\text { subject to } & \text { amplification gain } \Gamma(22) \\
& \text { target } n_{H}(26)-(27) \\
& \text { transient model }(7)-(12) .
\end{array}
$$

The Michaelis-Menten constant $\widetilde{\kappa}_{X^{*}}$ is set to $10^{-3}$ and the value of $\widetilde{\kappa}_{X}$ is then chosen in order to meet a desired ultrasensitivity target $n_{H} \in\{3,4,6,8,10,12,16\}$ (see figure $\mathrm{S} 2$ in the supporting material available at stacks.iop.org/PhysBio/9/045010/mmedia). The influence of the amplification and ultrasensitivity constraints on the minimal signaling times is considered in the operating regime $\rho^{S / X}=\rho^{P / X}=10^{-1}$ and for the kinetic parameters $\tilde{a}_{X}, \tilde{a}_{X^{*}}, \tilde{d}_{X}, \tilde{d}_{X^{*}}, \tilde{k}_{X}$ varying in the range $\left[10^{-3}, 10^{3}\right]$.

Figure 6 (left plots) displays the optimal signaling times as a function of the amplification gain $\Gamma$. The optimal rise time $\tau_{r}$ exhibits a non-monotonic relationship with the amplification gain and increases with the Hill coefficient target for a constant gain. In contrast, the ultrasensitivity requirement has a limited effect on the design for minimal decay time $\tau_{d}$. It is also found that the kinetic parameters $\tilde{a}_{X}$ and $\widetilde{a}_{X^{*}}$ stay at their upper bounds and $\widetilde{d}_{X^{*}}$ at its lower bound in all cases. On the other hand, the optimal values of the dissociation rate constant $\widetilde{d}_{X}$ of the kinase complex $X: S^{*}$ decrease significantly with increasing $n_{H}$, unlike those of the dissociation rate constant $\widetilde{k}_{X}$. This is attributed to the fact that high ultrasensitivity requires small values of the Michaelis-Menten constant $\widetilde{K}_{X}$-the value of the other Michaelis-Menten constant $\widetilde{K}_{X^{*}}$ being fixed at $10^{-3}$, which requires that $\widetilde{d}_{X} \ll \widetilde{a}_{X}-\widetilde{k}_{X}$ according to (25).

Perhaps the most striking finding from this inverse sensitivity analysis is that simple covalent modification cycles can be designed in such a way that they achieve high amplification and high ultrasensitivity, along with relatively short signaling times, on the order of 10 to 100 times the characteristic time $\left(\tilde{k}_{X}\right)^{-1}$ of the dissociation of the $X^{*}: P$ complex. It has often been postulated that multiple cycles in signaling cascades are needed to achieve multiple objectives [15], but interestingly, our results show that even a single interconvertible cycle can already meet several goals simultaneously.

\section{Designing flexible covalent modification cycles}

Due to the inherent variability of chemical reactions and cell components, the concentration levels in cells are subject to large fluctuations. This subsection addresses the question whether simple covalent modification cycles can be designed 
(a)

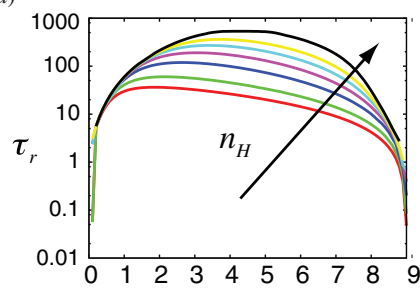

(b)

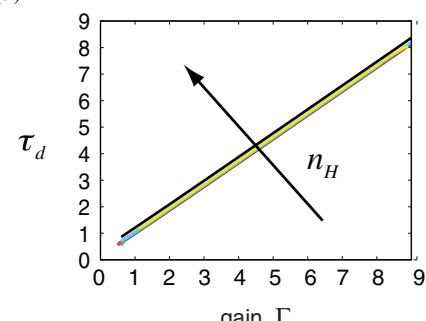

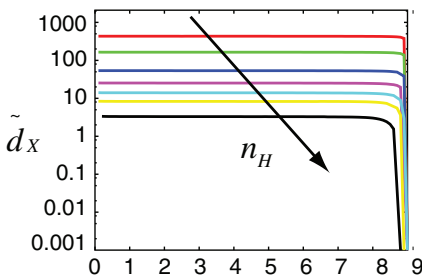

gain, $\Gamma$

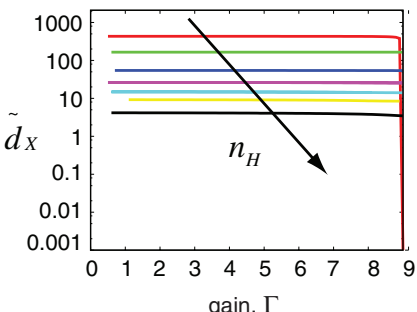

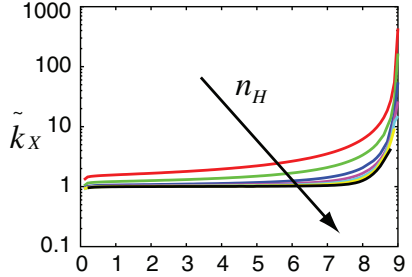

gain, $\Gamma$

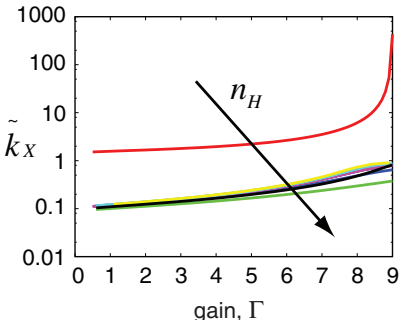

Figure 6. Design for minimal signaling rise time $\tau_{r}(a)$ and decay time $\tau_{d}(b)$ subject to given amplification and ultrasensitivity. The amplification gain $\Gamma$ is in the range $[0,9]$ and the Hill coefficient is in the set $n_{H} \in\{3,4,6,8,10,12,16\}$. The concentration ratios are $\rho^{S / X}=\rho^{P / X}=10^{-1}$.
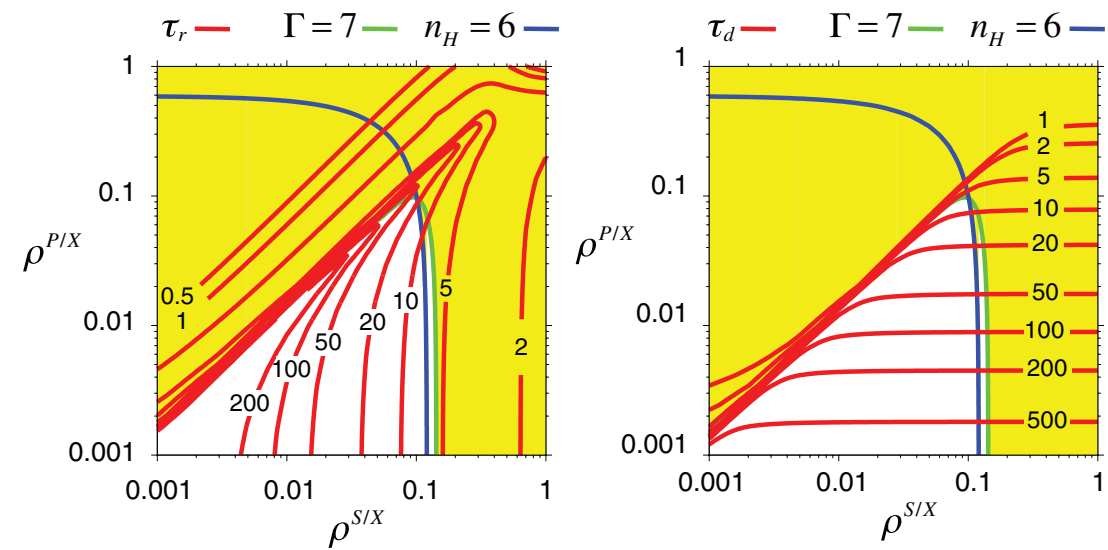

Figure 7. Flexibility of nominally designed cycle to change in the operating regime. The kinetic parameters are obtained from the solution of problems $\left(\mathrm{P}_{4}, \mathrm{P}_{5}\right)$ for nominal values of the concentration ratios as $\rho^{S / X}=\rho^{P / X}=10^{-1}$, and with $n_{H}=6$ and $\Gamma=7$. The functional characteristics are then monitored for actual $\rho^{S / X}$ and $\rho^{P / X}$ varying in the range $\left[10^{-3}, 1\right]$. The unshaded area comprises those values of $\rho^{S / X}$ and $\rho^{P / X}$ for which the amplification gain is larger than 7 , and the ultrasensitivity is larger than 6 . The labeled solid red lines correspond to the rise time (left plot) and decay time (right plot) contour lines.

so that multiple objectives are met simultaneously in spite of large variations in the enzyme-to-interconvertible-protein ratio $\rho^{S / X}$ and in the phosphatase-to-interconvertible-protein ratio $\rho^{P / X}$.

Flexibility of nominal design to variations in the operating regime. The robustness of a covalent modification cycle, optimized under nominal conditions as in the previous subsection, is investigated first. The results in figure 7 are for the parameter values

$\tilde{a}_{X}=\tilde{a}_{X^{*}}=10^{3}, \quad \tilde{d}_{X}=53, \quad \tilde{d}_{X^{*}}=10^{-3}, \quad \tilde{k}_{X}=1.42$, which minimize response time for amplification and ultrasensitivity objectives of $\Gamma=7$ and $n_{H}=6$, respectively, under the nominal regime $\rho^{S / X}=\rho^{P / X}=10^{-1}$ (see figure 6). Represented on these plots are all the other concentration regimes $\rho^{S / X}, \rho^{P / X}$ in the range $\left[10^{-3}, 1\right]$ for which the constraints $\Gamma \geqslant 7$ and $n_{H} \geqslant 6$ hold (unshaded area), together with the contour lines for the rise time objective (left plot) and the decay time objective (right plot).

Observe that the subset of operating regimes for which such a cycle achieves fast response times-for instance, both the rise time $\tau_{r}$ and the decay time $\tau_{d}$ less than 20 or 50 - corresponds to a rather narrow region around the nominal regime $\rho^{S / X}=\rho^{P / X}=10^{-1}$. Similar behavior was observed for different nominal regimes and with different amplification/ultrasensitivity thresholds (results not shown). This analysis clearly points out that a signaling cycle, which is designed to meet multiple objectives under a given nominal regime, may not be tolerant to large fluctuations around that particular regime. In other words, a nominally designed cycle would typically lack robustness, which arguably constitutes a strong barrier for their suitable implementation by living organisms. 

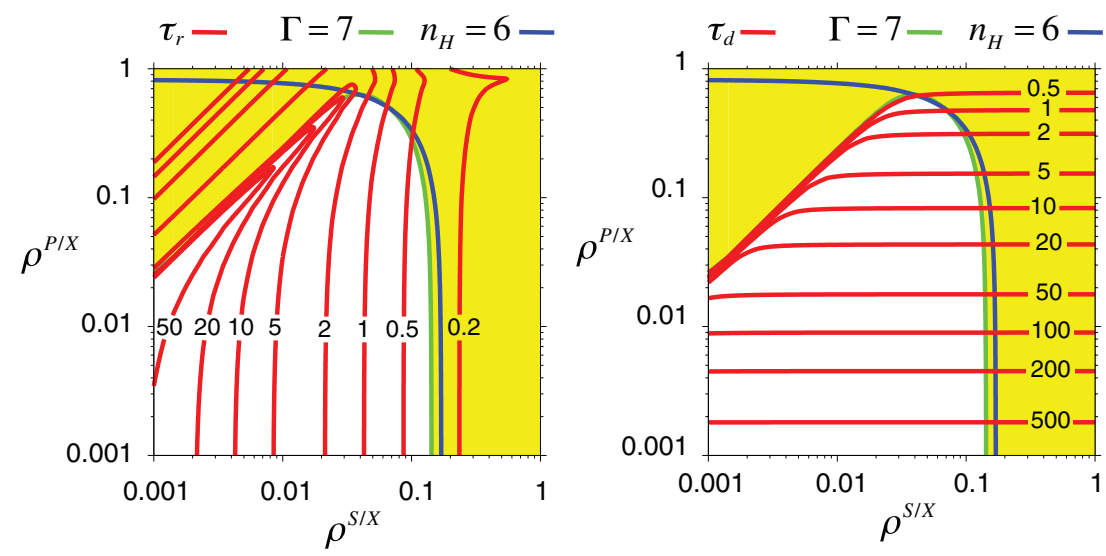

Figure 8. Flexibility of robustly designed cycle to change in the operating regime. The kinetic parameters are obtained from the solution of problem $\left(\mathrm{P}_{6}\right)$ for ranges of the concentration ratios as $\rho^{S / X}=10^{-1} \pm 50 \%, \rho^{P / X}=10^{-1}$, with the ultrasensitivity constraint $n_{H} \geqslant 6$ and the amplification constraint $\Gamma \geqslant 7$. All three functional characteristics are then monitored for that particular robust design when actual $\rho^{S / X}$ and $\rho^{P / X}$ vary in the range $\left[10^{-3}, 1\right]$. The unshaded area comprises those values of $\rho^{S / X}$ and $\rho^{P / X}$ for which the two constraints are satisfied. The labeled solid red lines correspond to the rise time (left plot) and decay time (right plot) contour lines.

Robust design to accommodate variations in the operating regime. To circumvent the lack of flexibility inherent to the nominal optimization problems $\left(\mathrm{P}_{4}, \mathrm{P}_{5}\right)$, the optimization formulation can be modified to explicitly account for the variability in concentration ratios $\rho^{S / X}, \rho^{P / X}$. In the field of optimization, such optimization problems fall into the scope of robust optimization [46, 47].

The robust optimization counterpart of problems $\left(\mathrm{P}_{4}, \mathrm{P}_{5}\right)$ formulates as follows:

find $\quad \tilde{a}_{X}, \widetilde{a}_{X^{*}}, \tilde{d}_{X}, \tilde{d}_{X^{*}}, \tilde{k}_{X}$ that

minimize expected signaling time $\mathrm{E}\left(\tau_{r}+\tau_{d}\right)$,

subject to lower bound on amplification gain,

$\Gamma \geqslant \Gamma^{L}$ for all $\rho^{S / X}, \rho^{P / X}$

lower bound on Hill coefficient,

$n_{H} \geqslant n_{H}^{L}$ for all $\rho^{S / X}, \rho^{P / X}$

transient model (7) $-(12)$.

steady-state model (A.3)-(A.8)

for the concentration ratios taking their values in a specified range $\rho^{S / X} \in\left[\rho^{S / X^{L}}, \rho^{S / X^{U}}\right]$ and $\rho^{P / X} \in\left[\rho^{P / X^{L}}, \rho^{P / X^{U}}\right]$. A uniform probability distribution is assumed here for $\rho^{S / X}$, $\rho^{P / X}$ over their entire ranges. The objective function in $\left(\mathrm{P}_{6}\right)$ is to minimize the expected value of the total signaling time $\left(\tau_{r}+\tau_{d}\right)$ over the range of $\rho^{S / X}, \rho^{P / X}$, rather than the total signaling time for a particular (nominal) regime as previously. Moreover, the inequality constraints on amplification and ultrasensitivity too must be satisfied over the entire range of $\rho^{S / X}, \rho^{P / X}$.

The results in figure 8 are for the parameter values

$$
\tilde{a}_{X}=\tilde{a}_{X^{*}}=10^{3}, \quad \tilde{d}_{X}=\tilde{d}_{X^{*}}=10^{-3}, \quad \tilde{k}_{X}=22.6,
$$

which minimize the expected total response time for amplification and ultrasensitivity thresholds of $\Gamma^{\mathrm{L}}=7$ and $n_{H}^{L}=6$, respectively, under the set of operating regimes $\rho^{S / X}=10^{-1} \pm 50 \%$ and $\rho^{P / X}=10^{-1}$. Represented on these plots are again all the other regimes $\rho^{S / X}, \rho^{P / X}$ in the range $\left[10^{-3}, 1\right]$ for which the constraints $\Gamma \geqslant 7$ and $n_{H} \geqslant 6$ hold (unshaded area), together with the contour lines for the rise time objective (left plot) and the decay time objective (right plot).
The subset of operating regimes for which such a robustly designed cycle achieves fast signaling times-e.g., both the rise time $\tau_{r}$ and the decay time $\tau_{d}$ less than 20 or 50 - is now much larger than that for the nominal design counterpart in figure 7 , spanning more than one order of magnitude in both $\rho^{S / X}$ and $\rho^{P / X}$. Equally remarkable is the clear decoupling between $\tau_{r}$ and $\rho^{P / X}$, on the one hand, and between $\tau_{d}$ and $\rho^{S / X}$, on the other hand. Such decoupling would, for instance, allow a pathway to maintain a fast signal rise time, while modulating its decay time by several orders of magnitude, and this simply by varying the phosphatase-to-interconvertible-protein ratio $\rho^{P / X}$ —arguably a very appealing feature that would enable flexibility and promote adaptedness in signaling cycles.

In a robustly designed cycle, the formation rates and the dissociation rates of the complexes $X: S^{*}$ and $X^{*}: P$ are pushed to their upper and lower bounds, respectively. Therefore, the only trade-off in the robust design lies in the choice of the kinetic parameter $\widetilde{k}_{X}$. It is found that a small value of $\widetilde{k}_{X}$ promotes higher ultrasensitivity, whereas a large value of $\widetilde{k}_{X}$ favors faster signaling times-both rise time and decay time-together with higher amplification gains. This trend is illustrated in figure S3 (see the supporting material available at stacks.iop.org/PhysBio/9/045010/mmedia), which depicts the same design objectives as in figure 8 , but in the case of a twofold increase in the kinetic parameter value as $\widetilde{k}_{X}$ $=50$. These results also confirm that the foregoing features of robustly designed cycles, including their flexibility, are conserved through a wide range of values for $\widetilde{k}_{X}$.

\section{Concluding remarks}

A particularly insightful finding of the analysis conducted in this paper is that, upon judicious selection of the kinetic parameters $\widetilde{a}_{X}, \widetilde{a}_{X^{*}}, \widetilde{d}_{X}, \widetilde{d}_{X^{*}}, \widetilde{k}_{X}$, a single covalent modification cycle not only has the ability to meet multiple objectives simultaneously, but that these objectives can also be made robust to large variations in the enzyme-to-interconvertibleprotein ratio $\rho^{S / X}$ and in the phosphatase-to-interconvertibleprotein ratio $\rho^{P / X}$. Another remarkable finding is that 
the responsiveness of a robustly designed cycle-both its rise time and decay time-can be modulated simply via increasing or decreasing $\rho^{S / X}$ and $\rho^{P / X}$, while maintaining high ultrasensitivity and amplification gains. Such features of signaling cycles give them the ability to perform various signaling functions robustly and make them versatile and adaptable. In particular, this analysis could help explain why signaling cycles are so ubiquitous in cell signaling.

This study also shows that optimization, and more specifically robust optimization, is particularly well suited for studying signaling pathways and generating hypotheses regarding their underlying design principles. Although focused on covalent modification cycles, there are no conceptual barriers toward the application of the same methodology to more complex signaling pathways, such as multi-level MAPK cascades or JAK-Stat pathways.

\section{Appendix. Mathematical formulations of dynamic optimization problems}

For the dynamic model, we use the compact notation

$$
\mathbf{F}(\xi(\tau), \dot{\xi}(\tau), p, r)=\mathbf{0},
$$

where the vector of state variables is $\xi:=$ $\left\{x^{*} \quad s \quad\left\{x: s^{*}\right\} \quad\left\{x^{*}: p\right\}\right\}^{T}$, the vector of kinetic parameters is $\mathbf{p}:=\left(\begin{array}{lllll}\widetilde{a}_{X} & \widetilde{a}_{X^{*}} & \widetilde{d}_{X} & \widetilde{d}_{X^{*}} & \widetilde{k}_{X}\end{array}\right)^{T}$ and the vector of concentration ratios is $\mathbf{r}:=\left(\begin{array}{ll}\rho^{S / X} & \rho^{P / X}\end{array}\right)^{T}$.

In the same manner, the steady-state model is represented as

$$
\mathbf{G}\left(\xi, \alpha_{X}, \mathbf{q}, \mathbf{r}\right)=\mathbf{0}
$$

with the vector of steady-state model parameters $\mathbf{q}:=$ $\left(\widetilde{K}_{X} \widetilde{K}_{X^{*}}\right)^{T}$.

In order to use a continuation approach [40], the steadystate equations (13)-(15) are differentiated with respect to $\alpha_{X}$,

$$
\begin{aligned}
& 0=\frac{\bullet}{\left\{x: s^{*}\right\}}\left(\alpha_{X}\right)+\frac{\widetilde{K}_{X}}{\left(\widetilde{K}_{X}+\bar{x}\right)^{2}}\left(\dot{\bullet} \bar{x}^{*}\left(\alpha_{X}\right)\right. \\
& \left.+\rho^{S / X} \frac{\bullet}{\left\{x: s^{*}\right\}}\left(\alpha_{X}\right)+\rho^{P / X} \frac{\bullet}{\left\{x^{*}: p\right\}}\left(\alpha_{X}\right)\right) \\
& 0=\overline{\left\{x^{*}: p\right\}}\left(\alpha_{X}\right)-\frac{\widetilde{K}_{X^{*}}}{\left(\widetilde{K}_{X^{*}}+\overline{x^{*}}\right)^{2}} \dot{\bullet} \overline{x^{*}}\left(\alpha_{X}\right) \\
& 0=\overline{\left\{x^{*}: p\right\}}\left(\alpha_{X}\right)-\alpha_{X} \overline{\left\{x: s^{*}\right\}}\left(\alpha_{X}\right)-\overline{\left\{x: s^{*}\right\}} \\
& 1=\bar{x}+\overline{x^{*}}+\rho^{S / X} \overline{\left\{x: s^{*}\right\}}+\rho^{P / X} \overline{\left\{x^{*}: p\right\}} \\
& 1=\overline{s^{*}}+\overline{\left\{x: s^{*}\right\}} \\
& 1=\bar{p}+\overline{\left\{x^{*}: p\right\}} \text {. }
\end{aligned}
$$

The mathematical formulation of the optimization problem $\left(\mathrm{P}_{1}\right)$, where the objective is to maximize ultrasensitivity subject to the steady-state model (A.3)-(A.8), reads

$$
\begin{array}{ll}
\max _{\mathbf{q}, \alpha_{X}^{10}, \alpha_{X}^{90}} & n_{H}=\frac{\ln (81)}{\ln \left(\alpha_{X}^{90}\right)-\left(\alpha_{X}^{10}\right)} \\
\text { subject to } & \frac{\partial \mathbf{G}}{\partial \xi} \dot{\xi}\left(\alpha_{X}\right)+\frac{\partial \mathbf{G}}{\partial \alpha_{X}}=\mathbf{0}, \\
& 0 \leqslant \alpha_{X} \leqslant \alpha_{X}^{\infty}, \mathbf{G}(\xi(0), 0, \mathbf{q}, \mathbf{r})=\mathbf{0}, \\
& x^{*}\left(\alpha_{X}^{10}\right)=0.1 x^{*}\left(\alpha_{X}^{\infty}\right), x^{*}\left(\alpha_{X}^{90}\right)=0.9 x^{*}\left(\alpha_{X}^{\infty}\right), \\
& 0 \leqslant \alpha_{X}^{10} \leqslant \alpha_{X}^{90} \leqslant \alpha_{X}^{\infty}, 10^{-2} \leqslant \mathbf{q} \leqslant 10^{2} .
\end{array}
$$

As noted, the initial conditions $\xi(0)$ are determined from $\mathbf{G}(\xi(0), 0, \mathbf{q}, \mathbf{r})=\mathbf{0}$. Equations (A.7) and (A.8) give $x^{*}(0)=$ $\left\{x^{*}: p\right\}(0)=0$ and $p(0)=1$; the remaining initial concentrations $x(0), s^{*}(0)$ and $\left\{x: s^{*}\right\}(0)$ depend on the kinetic constant $\widetilde{K}_{X}$ and the concentration ratio $\rho^{S / X}$ only and are given by

$$
\begin{gathered}
0=x(0)^{2}+\left(\widetilde{K}_{X}+\rho^{S / X}-1\right) x(0)-\widetilde{K}_{X} \\
\left\{x: s^{*}\right\}(0)=\frac{1-x(0)}{\rho^{S / X}} \\
s^{*}(0)=1-\frac{1-x(0)}{\rho^{S / X}} .
\end{gathered}
$$

The forward and backward step inputs in the transient studies require two different formulations of the fraction of inactive receptor as

$$
s:= \begin{cases}1, & \tau \leqslant 0 \\ 0, & \tau>0\end{cases}
$$

and

$$
s:= \begin{cases}0, & \tau \leqslant 0 \\ 1, & \tau>0 .\end{cases}
$$

Moreover, the activation/deactivation of the receptor is assumed to occur under very fast kinetics (i.e. $v=1000$ ) and is modeled as

$$
\frac{\mathrm{d} s}{\mathrm{~d} \tau}=-v s
$$

and

$$
\frac{\mathrm{d} s}{\mathrm{~d} \tau}=v s^{*} .
$$

We append (A.12) and (A.14) to the transient model when analyzing signaling rise time and (A.13) and (A.15) when analyzing signaling decay time.

The optimization problems $\left(\mathrm{P}_{2}\right)$ and $\left(\mathrm{P}_{3}\right)$ determine the kinetic parameter values that minimize the signaling times (the rise time $\tau_{r}$ and the decay time $\tau_{d}$, respectively), subject to a fixed amplification constraint $\Gamma$,

$$
\begin{array}{cl}
\min _{\mathbf{p}, \tau_{r}} & \tau_{r} \\
\text { subject to } & \mathbf{F}_{\mathbf{r}}(\xi(\tau), \dot{\xi}(\tau), \mathbf{p}, \mathbf{r})=\mathbf{0}, \\
& 0 \leqslant \tau \leqslant \tau^{\infty}, \xi(0)=\xi_{r_{0}}, \\
& x^{*}\left(\tau_{r}\right)-0.9 x^{*}\left(\tau^{\infty}\right)=0, \\
& \Gamma \rho^{S / X}-x^{*}\left(\tau^{\infty}\right)=0, \\
& 10^{-3} \leqslant \mathbf{p} \leqslant 10^{3},
\end{array}
$$

and

$$
\begin{array}{ll}
\min _{\mathbf{p}, \tau_{d}} & \tau_{d} \\
\text { subject to } & \mathbf{F}_{\mathbf{d}}(\xi(\tau), \dot{\xi}(\tau), \mathbf{p}, \mathbf{r})=\mathbf{0}, \\
& 0 \leqslant \tau \leqslant \tau^{\infty}, \xi(0)=\xi_{d_{0}}, \\
& x^{*}\left(\tau_{d}\right)-0.1 x^{*}\left(\tau^{\infty}\right)=0, \\
& \Gamma \rho^{S / X}-x^{*}(0)=0, \\
& 10^{-3} \leqslant \mathbf{p} \leqslant 10^{3},
\end{array}
$$

The previous optimization formulations are then extended to include additional constraints. The goal of the resulting optimization problems $\left(\mathrm{P}_{4}\right)$ and $\left(\mathrm{P}_{5}\right)$ is to determine the kinetic 
parameter values that minimize the signaling times, subject to given amplification and ultrasensitivity thresholds,

$$
\begin{array}{ll}
\min _{\mathbf{p}, \tau_{r}} & \tau_{r} \\
\text { subject to } & \mathbf{F}_{\mathbf{r}}(\xi(\tau), \dot{\xi}(\tau), \mathbf{p}, \mathbf{r})=\mathbf{0}, \\
& 0 \leqslant \tau \leqslant \tau^{\infty}, \xi(0)=\xi_{r_{0}}, \\
& x^{*}\left(\tau_{r}\right)-0.9 x^{*}\left(\tau^{\infty}\right)=0, \\
& \Gamma \rho^{S / X}-x^{*}\left(\tau^{\infty}\right)=0, \\
& \widetilde{d}_{X}+\widetilde{k}_{X}-\widetilde{\kappa}_{X} \widetilde{a}_{X}=0, \\
& \widetilde{d}_{X^{*}}+1-\widetilde{\kappa}_{X^{*}} \widetilde{a}_{X^{*}}=0, \\
& 10^{-3} \leqslant \mathbf{p} \leqslant 10^{3} .
\end{array}
$$

and

$$
\begin{array}{ll}
\min _{\mathbf{p}, \tau_{d}} & \tau_{d} \\
\text { subject to } & \mathbf{F}_{\mathbf{d}}(\xi(\tau), \dot{\xi}(\tau), \mathbf{p}, \mathbf{r})=\mathbf{0}, \\
& 0 \leqslant \tau \leqslant \tau^{\infty}, \xi(0)=\xi_{d_{0}}, \\
& x^{*}\left(\tau_{d}\right)-0.1 x^{*}\left(\tau^{\infty}\right)=0, \\
& \Gamma \rho^{S / X}-x^{*}(0)=0, \\
& \widetilde{d}_{X}+\widetilde{k}_{X}-\widetilde{\kappa}_{X} \widetilde{a}_{X}=0, \\
& \widetilde{d}_{X^{*}}+1-\widetilde{\kappa}_{X^{*}} \widetilde{a}_{X^{*}}=0, \\
& 10^{-3} \leqslant \mathbf{p} \leqslant 10^{3} .
\end{array}
$$

To account for the inherent variability of the concentration ratios $\mathbf{r}$, we use a robust optimization framework. The optimization problem $\left(\mathrm{P}_{6}\right)$ determines the kinetic parameter values that minimize the expected signal propagation times, subject to lower bounds on amplification and ultrasensitivity,

$$
\begin{array}{ll}
\min _{\mathbf{p}, \tau_{r}, \tau_{d}, \mathbf{q}, \alpha_{X}^{10}, \alpha_{X}^{90}} & \int_{\mathbf{r}^{L}}^{\mathbf{r}^{U}}\left[\tau_{r}(\mathbf{r})+\tau_{d}(\mathbf{r})\right] \mathrm{d} \mathbf{r} \\
\text { subject to } & \mathbf{F}_{\mathbf{r}}(\xi(\tau), \dot{\xi}(\tau), \mathbf{p}, \mathbf{r})=\mathbf{0}, \\
& 0 \leqslant \tau \leqslant \tau^{\infty}, \xi(0)=\mathrm{P}_{r_{0}}, \\
& x_{r}^{*}\left(\tau_{r}\right)-0.9 x_{r}^{*}\left(\tau^{\infty}\right)=0, \\
& \mathbf{F}_{\mathbf{d}}(\xi(\tau), \dot{\xi}(\tau), \mathbf{p}, \mathbf{r})=\mathbf{0}, \\
& 0 \leqslant \tau \leqslant \tau^{\infty}, \xi(0)=\xi_{d_{0}}, \\
& x_{d}^{*}\left(\tau_{d}\right)-0.1 x_{d}^{*}\left(\tau^{\infty}\right)=0, \\
& \frac{x_{r}^{*}\left(\tau^{\infty}\right)}{\rho^{S / X} \geqslant \Gamma^{L}, \forall \mathbf{r} \in\left[\mathbf{r}^{L}, \mathbf{r}^{U}\right],} \\
& \frac{\partial \mathbf{G}}{\partial \xi}\left(\alpha_{X}\right)+\frac{\partial \mathbf{G}}{\partial \alpha_{X}}=\mathbf{0}, \\
& 0 \leqslant \alpha_{X} \leqslant \alpha_{X}^{\infty}, \mathbf{G}(\xi(0), 0, \mathbf{q}, \mathbf{r})=\mathbf{0} \\
& x^{*}\left(\alpha_{X}^{10}\right)=0.1 x^{*}\left(\alpha_{X}^{\infty}\right), x^{*}\left(\alpha_{X}^{90}\right)=0.9 x^{*}\left(\alpha_{X}^{\infty}\right), \\
& \tilde{K}_{X}=\frac{\tilde{d}_{X}+\tilde{k}_{X}}{\tilde{a}_{X}}, \tilde{K}_{X^{*}}=\frac{\tilde{d}_{X^{*}}+1}{\tilde{a}_{X^{*}}}, \\
& \frac{\ln (81)}{\ln \left(\alpha_{X}^{90}\right)-\ln \left(\alpha_{X}^{90}\right)} \geqslant n_{H}^{L}, \forall \mathbf{r} \in\left[\mathbf{r}^{L}, \mathbf{r}^{U}\right], \\
& 0 \leqslant \tau_{r}, \tau_{d} \leqslant \tau^{\infty}, 0 \leqslant \alpha_{X}^{10} \leqslant \alpha_{X}^{90} \leqslant \alpha_{X}^{\infty}, \\
& 10^{-3} \leqslant \mathbf{p} \leqslant 10^{3}, 10^{-2} \leqslant \mathbf{q} \leqslant 10^{2} .
\end{array}
$$

Problems $\left(\mathrm{P}_{1}\right)-\left(\mathrm{P}_{5}\right)$ are dynamic optimization problems. The goal of dynamic optimization (DO) is to find the optimal input parameters and/or profiles of a dynamic system. Optimality is defined as the minimization or maximization of an objective function, subject to specified constraints. Part of these constraints are given in the form of differential equations.

We solve these problems using the sequential method of dynamic optimization. The NLP subproblems are solved with the SQP solver SNOPT [48]. We have used the solver
DSL48S [49], which is part of DAEPACK [50] and for solving the initial-value problems (IVPs) in DAEs. This package is well suited for large-scale problems. DAEPACK is also used for consistent initialization and for calculating first-order parametric sensitivities. Finally, all the necessary differentiations (of the models, the objective functions and the constraint functions) are generated using the capabilities of DAEPACK. In order to avoid local solutions that can be very misleading, we systematically perform the optimization from multiple randomly generated starting points.

Problem $\left(\mathrm{P}_{6}\right)$ is a robust dynamic optimization problem. The goal of robust optimization (RO) is to search for designs and solutions that are immune to the effect of parametric uncertainty.

We solve this problem by using the so-called DAEs inside DAE approach, where the inner set of DAEs describes the system and the outer set describes the uncertainty [51].

\section{References}

[1] Widmann C, Gibson S, Jarpe M B and Johnson G L 1999 Mitogen-activated protein kinase: conservation of a three-kinase module from yeast to human Physiol. Rev. 79 143-80

[2] Sebolt-Leopold J S and Herrera R 2004 Targeting the mitogen-activated protein kinase cascade to treat cancer Nature Rev. Cancer 4 937-47

[3] Vayttaden S J, Ajay S M and Bhalla U S 2004 A spectrum of models of signaling pathways Chem. Biochem. 5 1365-74

[4] Klipp E and Liebermeister W 2006 Mathematical modeling of intracellular signaling pathways BMC Neurosci. 7 S10

[5] Kiel C, Yus E and Serrano L 2010 Engineering signal transduction pathways Cell 140 33-47

[6] Goldbeter A and Koshland D E Jr 1981 An amplified sensitivity arising from covalent modification in biological systems Proc. Natl Acad. Sci. USA 78 6840-4

[7] Huang C Y and Ferrell J E Jr 1996 Ultrasensitivity in the mitogen-activated protein kinase cascade Proc. Natl Acad. Sci. USA 93 10078-83

[8] Bhalla U and Iyengar R 1999 Emergent properties of networks of biological signaling pathways Science 283 381-7

[9] Ferrell J E Jr 2002 Self-perpetuating states in signal transduction: positive feedback, double-negative feedback and bistability Curr. Opin. Cell. Biol. 14 140-8

[10] Angeli D, Ferrell J E Jr and Sontag E D 2004 Detection of multistability, bifurcations, and hysteresis in a large class of biological positive-feed back systems Proc. Natl Acad. Sci. USA 101 1822-7

[11] Markevich N I, Hoek J B and Kholodenko B N 2004 Signaling switches and bistability arising from multisite phosphorylation in protein kinase cascades J. Cell Biol. $164353-9$

[12] Legewie S, Schoeberl B, Blüthgen N and Herzel H 2007 Competing docking interactions can bring about bistability in the MAPK cascade Biophys. J. 93 2279-88

[13] Kholodenko B N 2000 Negative feedback and ultrasensitivity can bring about oscillations in the mitogen-activated protein kinase cascades Eur. J. Biochem. 267 1583-8

[14] Qiao L, Nachbar R B, Kevrekidis I G and Shvartsman S Y 2007 Bistability and oscillations in the Huang-Ferrell model of MAPK signaling PLoS Comput. Biol. 3 1819-26

[15] Heinrich R, Neel B G and Rapoport T A 2002 Mathematical models of protein kinase signal transduction Mol. Cell. 9 957-70 
[16] Chapman S and Asthagiri A R 2004 Resistance to signal activation governs design features of the MAP kinase signaling module Biotechnol. Bioeng. 85 311-22

[17] Hornberg J J, Bruggeman F J, Binder B, Geest C R, Bij de Vaate A J M, Lankelma J, Heinrich R and Westerhoff H V 2005 Principles behind the multifarious control of signal transduction-ERK phosphorylation and kinase/phosphatase control FEBS J. 272 244-58

[18] Gomez-Uribe C, Verghese G C and Mirny L A 2007 Operating regimes of signaling cycle: statics, dynamics, and noise filtering PLoS Comput. Biol. 3 2487-97

[19] Ventura A C, Sepulchre J A and Merajver S D 2008 A hidden feedback in signaling cascades is revealed PLoS Comput. Biol. 4 1-14

[20] Shvartsman S Y, Coppey M and Berezhkovskii A M 2009 MAPK signaling in equations and embryos Fly 3 62-7

[21] Saltelli A, Ratto M, Andres T, Campolongo F, Cariboni J, Gatelli D, Saisana M and Tarantola S 2008 Global Sensitivity Analysis: The Primer (Chichester: Wiley)

[22] Nocedal J and Wright S J 1999 Numerical Optimization (New York: Springer)

[23] Zak D E, Stelling J and Doyle F J III 2005 Sensitivity analysis of oscillatory (bio)chemical systems Comput. Chem. Eng. 29 663-73

[24] Schwacke J H and Voit E O 2005 Computation and analysis of time-dependent sensitivities in generalized mass action systems J. Theor. Biol. 236 21-38

[25] Zi Z, Cho K H, Sung M H, Xia X, Zheng J and Sun Z 2005 In silico identification of the key components and steps in IFN- $\gamma$ induced JAK-STAT signaling pathway FEBS Lett. 579 1101-8

[26] van Riel N A W 2006 Dynamic modelling and analysis of biochemical networks: mechanism-based models and model-based experiments Briefings Bioinform. 7 364-74

[27] Yue H, Brown M, Knowles J, Wang H, Broomhead D S and Kell D B 2006 Insights into the behaviour of systems biology models from dynamic sensitivity and identifiability analysis: a case study of an NF- $\kappa$ B signalling pathway Mol. Biosyst. 2 640-9

[28] Chen W W, Schoeberl B, Jasper P J, Niepel M, Nielsen U B, Lauffenburger D A and Sorger P K 2009 Input-output behavior of ErbB signaling pathways as revealed by a mass action model trained against dynamic data Mol. Syst. Biol. 5239

[29] Heinrich R, Schuster S and Holzhutter H G 1991 Mathematical analysis of enzymic reaction systems using optimization principles Eur. J. Biochem. 201 1-21

[30] Kikuchi S 2003 Dynamic modeling of genetic networks using genetic algorithm and S-system Bioinformatics 19 643-50

[31] Banga J R 2008 Optimization in computational systems biology BMC Syst. Biol. 247

[32] Goh C J and Teo K L 1988 Control parametrization: a unified approach to optimal control problem with general constraints Automatica 24 3-18

[33] Biegler L T, Cervantes A and Waechter A 2002 Advances in simultaneous strategies for dynamic process optimization Chem. Eng. Sci. 57 575-93
[34] Adiwijaya B S, Barton P I and Tidor B 2006 Biological network design strategies: discovery through dynamic optimization Mol. Biosyst. 2 650-9

[35] Rieger T R 2005 Mathematical modeling of the eukaryotic heat shock response and associated ultrasensitive signaling cascades PhD Thesis Northwestern University, Evanston, IL

[36] Blüthgen N, Bruggeman F J, Legewie S, Herzel H, Westerhoff H V and Kholodenko B N 2006 Effects of sequestration on signal transduction cascades FEBS $J$. 273 895-906

[37] Millat T, Bullinger E, Rohwer J and Wolkenhauer O 2007 Approximations and their consequences for dynamic modelling of signal transduction pathways Math. Biosci. $20740-57$

[38] Liu X, Bardwell L and Nie Q 2010 A combination of multisite phosphorylation and substrate sequestration produces switchlike responses Biophys. J. 98 1396-407

[39] Thattai M and van Oudenaarden A 2002 Attenuation of noise in ultrasensitive signaling cascades Biophys. $J$. 82 2943-50

[40] Allgower E L and Georg K 2003 Introduction to Numerical Continuation Methods (Philadelphia, PA: SIAM)

[41] Park T and Barton P I 1996 State event location in differential-algebraic models ACM Trans. Model. Comput. Simul. 6 137-65

[42] Asthagiri A R and Lauffenburger D A 2001 A computational study of feedback effects on signal dynamics in a mitogen-activated protein kinase (MAPK) pathway model Biotechnol. Prog. 17 227-39

[43] Ferrell J E Jr 1996 Tripping the switch fantastic: how a protein kinase cascade can convert graded inputs into switch-like outputs Trends Biochem. Sci. 21 460-6

[44] Legewie S, Blüthgen N and Herzel H 2005 Quantitative analysis of ultrasensitive responses FEBS J. 272 4071-9

[45] Buchler N E and Cross F R 2009 Protein sequestration generates a flexible ultrasensitive response in a genetic network Mol. Syst. Biol. 5272

[46] Zhang Y 2007 General robust-optimization formulation for nonlinear programming J. Optim. Theory Appl. $132111-24$

[47] Diehl M, Gerhard J, Marquardt W and Moenigmann M 2008 Numerical solution approaches for robust nonlinear optimal control problems Comput. Chem. Eng. 32 1287-300

[48] Gill P E, Murray W and Saunders M A 2005 SNOPT: an SQP algorithm for large-scale constrained optimization SIAM Rev. 48 99-131

[49] Feehery W F, Tolsma J E and Barton P I 1997 Efficient sensitivity analysis of large-scale differential-algebraic systems Appl. Numer. Math. 25 41-54

[50] Tolsma J E and Barton P I 2000 DAEPACK: an open modeling environment for legacy models Ind. Eng. Chem. Res. 39 1826-39

[51] Chachuat B and Barton P I 2007 Numerical simulation of a class of PDAEs with a separation of time scales Progress In Industrial Mathematics at ECMI 2006, Mathematics in Industry ed L L Bonilla et al (Berlin: Springer) vol 12 pp 512-7 